\title{
Author Correction: High-precision digital terahertz phase manipulation within a multichannel field perturbation coding chip
}

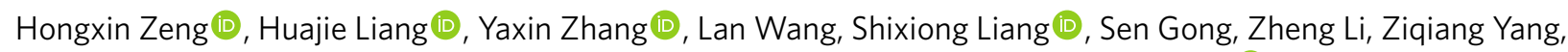
Xilin Zhang, Feng Lan, Zhihong Feng, Yubin Gong, Ziqiang Yang and Daniel M. Mittleman (D)

Correction to: Nature Photonics, https://doi.org/10.1038/s41566-021-00851-6, published online 9 August 2021.

In the version of this Article initially published, there was an omission and an error in author initials in the Acknowledgements section. The correct statement should now read:

“This work was supported by...the National Natural Science Foundation of China under contracts 61921002 (Y.G.) and U20A20212 (Y.Z.)."

The errors have been corrected in the online version of the Article.

Published: 31 August 2021

https://doi.org/10.1038/s41566-021-00874-Z

๑) Springer Nature Limited 2021

\section{Publisher Correction: Ultra-coherent Fano laser based on a bound state in the continuum}

Yi Yu (D), Aurimas Sakanas, Aref Rasoulzadeh Zali (D), Elizaveta Semenova (D), Kresten Yvind (1) and Jesper Mørk (1)

Correction to: Nature Photonics https://doi.org/10.1038/s41566-021-00860-5, published online 12 August 2021.

In the version of this article initially published, in Fig. 1d, the red rectangle indicating buried heterostructure gain region appeared in the center of the wave guide rather than at the far left. This red bar now been shifted left. Additionally, in the last sentence of the Discussion, a typo ("Fano-Perot") has now been fixed to correctly read "Fabry-Perot type BIC."

The original article has been corrected in the online version of the paper.

Published online: 26 August 2021

https://doi.org/10.1038/s41566-021-00883-y

๑ The Author(s), under exclusive licence to Springer Nature Limited 2021 